\title{
FabR regulates Salmonella biofilm formation via its direct target FabB
}

\author{
Kim Hermans ${ }^{1}$, Stefanie Roberfroid ${ }^{1}$, Inge M. Thijs ${ }^{1}$, Gwendoline Kint ${ }^{1}$, David De Coster ${ }^{1}$, Kathleen Marchal', \\ Jos Vanderleyden ${ }^{1}$, Sigrid C. J. De Keersmaecker ${ }^{1,2}$ and Hans P. Steenackers ${ }^{1 *}$
}

\begin{abstract}
Background: Biofilm formation is an important survival strategy of Salmonella in all environments. By mutant screening, we showed a knock-out mutant of fabR, encoding a repressor of unsaturated fatty acid biosynthesis (UFA), to have impaired biofilm formation. In order to unravel how this regulator impinges on Salmonella biofilm formation, we aimed at elucidating the $S$. Typhimurium FabR regulon. Hereto, we applied a combinatorial highthroughput approach, combining ChIP-chip with transcriptomics.

Results: All the previously identified E. coli FabR transcriptional target genes ( $f a b A$, fabB and yqfA) were shown to be direct $S$. Typhimurium FabR targets as well. As we found a fabB overexpressing strain to partly mimic the biofilm defect of the fabR mutant, the effect of FabR on biofilms can be attributed at least partly to FabB, which plays a key role in UFA biosynthesis. Additionally, ChIP-chip identified a number of novel direct FabR targets (the intergenic regions between $h p a R / h p a G$ and $d d g / y d f Z$ ) and yet putative direct targets (i.a. genes involved in tRNA metabolism, ribosome synthesis and translation). Next to UFA biosynthesis, a number of these direct targets and other indirect targets identified by transcriptomics (e.g. ribosomal genes, ompA, ompC, ompX, osmB, osmC, ssel), could possibly contribute to the effect of FabR on biofilm formation.
\end{abstract}

Conclusion: Overall, our results point at the importance of FabR and UFA biosynthesis in Salmonella biofilm formation and their role as potential targets for biofilm inhibitory strategies.

Keywords: Salmonella, Biofilm, FabR, FabB, Unsaturated fatty acids, ChIP-chip

\section{Background}

Bacteria predominantly grow inside multicellular communities attached to solid surfaces and enclosed in a self-produced polymeric matrix, called biofilms [1]. In fact, it was shown that the majority of all bacterial infections are related to biofilm growth, stressing the importance of this life style [2]. Because of the protected environment, bacteria within biofilms are less sensitive to environmental stresses, including disinfectants and antibiotics, and are as a consequence very difficult to eradicate [3]. Pathogens like Salmonella are able to survive in biofilms on biotic as well as abiotic surfaces [4] as was shown for i.a. plastic, stainless steel, plant surfaces and gallstones [3, 5-7].

\footnotetext{
* Correspondence: Hans.Steenackers@biw.kuleuven.be

${ }^{1}$ Department of Microbial and Molecular Systems, Centre of Microbial and Plant Genetics, Katholieke Universiteit Leuven, Kasteelpark Arenberg 20, 3001 Leuven, Belgium

Full list of author information is available at the end of the article
}

The extracellular matrix of Salmonella biofilms contains a variety of proteinaceous compounds and exopolysaccharides, including curli fimbriae and cellulose [8]. Furthermore, the presence of flagella and fatty acid containing structures such as lipopolysaccharides was shown to be important in Salmonella biofilms [7]. However, the exact composition of the matrix and the appearing ratios of the different structures are highly dependent on the environmental conditions in the used biofilm set-up [9]. It was shown for example that an incomplete LPS fraction does not affect biofilm formation capacity of Salmonella on hydrophobic gallstone surfaces, but highly reduces its biofilm capacity on hydrophilic glass surfaces [7].

Synthesis of all these structures is strongly regulated as the regulatory networks inside biofilms as well as metabolism are highly complex [4]. The central transcription regulator in Salmonella biofilm formation metabolism is CsgD, which positively regulates the 
production of curli and cellulose in the extracellular biofilm matrix $[10,11]$. This regulator itself shows an enhanced expression in the presence of high c-di-GMP concentrations [12], a secondary messenger molecule which has been studied extensively in regulation of bacterial multicellular behavior, motility and virulence [13]. Knowledge about the complex regulatory processes in biofilm formation can provide more insight into survival strategies of $S$. Typhimurium in non-host environments and can be the fundament of new eradication methods.

Bacteria strictly regulate their cellular membrane composition in response to changes of environmental conditions, in order to adjust membrane fluidity and optimize associated membrane functions [14]. Variations in growth temperature [15-18], $\mathrm{pH}[16,19,20]$, ethanol concentration [21] and external osmolality [22], as well as transition to the stationary phase [23] have been shown to lead to changes in membrane fatty acid composition. These changes in membrane fatty acid composition and membrane fluidity have been shown to affect bacterial thermotolerance [24, 25], acid resistance [26] and pressure resistance [27] .

In Escherichia coli, a mechanism was discovered that controls the ratio of mono-unsaturated fatty acids (UFA) compared to saturated fatty acids (SFA) present in the membrane in response to the composition of the cellular pool of long chain acyl-thioesters. [28]. A central transcription regulator in this process is FabR (also called YijC) which, in response to mono-unsaturated fatty acid thioesters, possesses an enhanced affinity for binding to the promoter sequence of $f a b B$ and in lesser extent to the $f a b A$ promoter. This promoter binding represses transcription of these $f a b$ genes which are involved in UFA biosynthesis [29]. FabA introduces the double bond into the growing acyl chain by catalyzing dehydration of $\beta$-hydroxydecanoyl-ACP and the isomerization of the resulting product to cis-3-decenoyl-ACP [29]. FabB elongates cis-3-decenoyl-ACP to cis-5-dodecenoyl- ACP, which enters the standard fatty acid synthesis cycle and becomes elongated to the 16- and 18-carbon UFAs [30]. Conversely, another transcription regulator, FadR, stimulates UFA biosynthesis by binding to the $f a b A$ end $f a b B$ promoter. This activation is relieved by dissociation of FadR from the $f a b A$ and $f a b B$ promoter regions in response to long-chain fatty acids [31]. Together these transcription regulators ensure a wellbalanced ratio of SFA compared to UFA and as such sustaining the biophysical properties of the cell membrane phospholipids that are of great importance for bacterial growth and survival.

By mutant screening, we found a $f a b R$ knock-out mutant of Salmonella enterica serovar Typhimurium to have an impaired biofilm formation. To get more insight into the way FabR regulates Salmonella biofilm formation, we mapped the full $S$. Typhimurium SL1344 FabR regulon and identified its direct and indirect target genes. Hereto, we combined chromatin immunoprecipitation (ChIP) coupled with $S$. Typhimurium wholegenome tiling arrays (ChIP-chip) and transcriptomics comparing gene expression in a $f a b R$ deletion mutant and wildtype $S$. Typhimurium SL1344. ChIP-chip facilitates the identification of direct regulatory targets on a genome-wide scale in vivo [32] and does not rely on in vitro observations (as most biochemical methods) or the sometimes spurious presence of consensus DNAbinding sites (as in in silico motif detection algorithms). Combining it with a transcriptomics approach allows the discrimination between direct and indirect target genes and reduces its inherent noise [33-36] This provided the first evidence for the direct repression of $f a b A, f a b B$ and yqfA expression by FabR in $S$. Typhimurium, confirming current knowledge generated in E. coli. We showed that $f a b B$ overexpression results in a decreased biofilm formation, indicating a role for FabB (involved in UFA biosynthesis) in mediating the effect of fabR on biofilm formation. Altered UFA synthesis might impact on biofilm formation in several ways, either by alterations in membrane fatty acid composition, membrane fluidity and surface properties or by a role of free UFA's as signaling molecules. Moreover, next to UFA biosynthesis, a number of other genes, known to be involved in biofilm formation, identified to be (in)directly regulated by FabR (e.g. ribosomal genes, ompA, ompC, ompX, osmB, osm C, sseI), could possibly contribute to the effect of FabR on biofilm formation.

\section{Methods}

\section{Bacterial strains, plasmids and media}

All strains and plasmids used in this study are listed in Table 1. Salmonella cultures were routinely grown with aeration in Luria-Bertani (LB) broth, on LB agar plates containing $15 \mathrm{~g} / \mathrm{l}$ agar (Invitrogen) or in tryptic soy broth (BD Biosciences, $30 \mathrm{~g} / \mathrm{l}$ ) diluted 1/20 (TSB $1 / 20$ ), with the addition of antibiotics (ampicillin (Ap), $100 \mu \mathrm{g} / \mathrm{ml}$; chloramphenicol (Cm), $25 \mu \mathrm{g} / \mathrm{ml})$, if appropriate.

Standard protocols were used for molecular cloning [37]. Cloning steps were performed using E. coli DH5 $\alpha$ and TOP10F' and the final, constructed plasmids were electroporated to the $S$. Typhimurium SL1344 strains using a Bio-Rad gene pulser. Restriction enzymes were purchased from New England Biolabs and used according to the manufacturer's instructions. All primers used and their purposes are listed in Additional file 1: Table S2. The sequences used for primer construction were obtained from the complete genome sequence of $S$. Typhimurium SL1344, as available via the website of the Sanger 
Table 1 Bacterial strains and plasmids

\begin{tabular}{|c|c|c|}
\hline Name & Description & Reference \\
\hline \multicolumn{3}{|l|}{ Strains } \\
\hline E. coli DH5a & $\begin{array}{l}\mathrm{F}^{-} \phi 80 \Delta \operatorname{lacZM15} \triangle(\text { lacZYAargF)U169 deoP recA1 endA1 } \\
\left.\text { hsdR17( (r- } \mathrm{m}_{\mathrm{k}}^{-}\right) \lambda^{-}\end{array}$ & Gibco BRL \\
\hline E. coli TOP10F' & 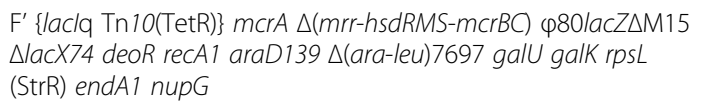 & Invitrogen \\
\hline S. Typhimurium SL1344 & Wildtype strain, xyl hisG rpsL; virulent; $\mathrm{Sm}^{\mathrm{R}}$ & [82] \\
\hline CMPG5624 & S. Typhimurium SL1344, $\triangle f a b R$ & This study \\
\hline CMPG5825 & S. Typhimurium SL1344, fabR-M9 & This study \\
\hline \multicolumn{3}{|l|}{ Plasmids } \\
\hline c3390 & Spel-myc9-Spel cassette in pBlueskript SKII- & [39] \\
\hline pCP20 & flp, ts-rep-[cil857] $(\lambda)$ ts, $\mathrm{Ap}^{\mathrm{R}}, \mathrm{Cm}^{\mathrm{R}}$ & [38] \\
\hline pCMPG5553 & yqfA cloned Xbal/Sacl in pFAJ1708 & This study \\
\hline pCMPG5678 & fabR cloned EcoRI/BamHI in pFAJ1708 & This study \\
\hline pCMPG10118 & fabA cloned Xbal/EcoRl in pFAJ1708 & This study \\
\hline pCMPG10119 & fabB cloned Xbal/EcoRl in pFAJ1708 & This study \\
\hline pFPV25 & $\begin{array}{l}\text { Promoter-trap vector constructed by inserting an EcoRI-Hindlll } \\
\text { fragment containing a promoterless gfpmut3 [83] into plasmid } \\
\text { pED350 (colE1, bla, mob) [84]; ApR }\end{array}$ & {$[85]$} \\
\hline pKD3 & $\begin{array}{l}\text { Plasmid used as template for construction of Salmonella } \\
\text { mutants; } \mathrm{Ap}^{R}, \mathrm{Cm}^{R}\end{array}$ & [38] \\
\hline pKD46 & Lambda Red helper plasmid, $A p^{R}$ & [38] \\
\hline
\end{tabular}

Institute (U.K.), (http://www.sanger.ac.uk/Projects/Salmonella). Plasmids pCMPG5678, pCMPG5553, pCMPG10118 and pCMPG10119 were constructed by cloning respectively the PCR-amplified fabR (STM4127), yqfA (STM3049), $f a b A$ (STM1067) and $f a b B$ (STM2378) coding sequences, as EcoRI/BamHI $(f a b R), \mathrm{XbaI} / \mathrm{SacI}(y q f A)$ or $\mathrm{XbaI} / \mathrm{EcoRI}$ $(f a b A$ and $f a b B)$ fragments, downstream of the constitutive nptII promoter into the RK2 based plasmid pFAJ1708. The S. Typhimurium SL1344 $\triangle$ fabR (CMPG5624) mutant was constructed using the procedure described by Datsenko and Wanner [38], starting from plasmid pKD3. A strain with a chromosomally encoded 9xMyc epitope-tagged FabR was constructed as previously described [35], using primers PRO379, PRO254, PRO494, PRO495, pCRII TOPO (Invitrogen), c3390 [39]. All strains and constructs were finally verified by PCR and sequencing analysis.

\section{Phenotypic assays}

Two different biofilm assays were used: (i) biofilms were formed using the static high-throughput peg system and at the bottom of petri dishes at 16 or $25^{\circ} \mathrm{C}$ in TSB $1 / 20$ for $48 \mathrm{~h}$, as previously described [40]. The only modification being that 150 instead of $200 \mu \mathrm{l}$ was added to each well of the microtiter plate. For biofilm formation studies at $30{ }^{\circ} \mathrm{C}$, the high-throughput peg system was incubated in a humid environment to minimize evaporation from the wells. To test the effect of free fatty acids on biofilm formation, two-fold serial dilutions of the fatty acids were prepared in the peg system as described previously [41]. Results of the peg-based assays are shown in the figures as a percentage of biofilm formed, compared to wildtype S. Typhimurium strain SL1344 (100\%) and error bars represent the standard deviation of at least three independent measurements. (ii) Biofilm formation on the bottom of small polystyrene petri dishes (60 mm diameter, Greiner Bio-One) was performed by adding $10 \mathrm{ml}$ of a 1:100 dilution of the particular S. Typhimurium strain into TSB $1 / 20$ broth. After 48 h stationary incubation at $25{ }^{\circ} \mathrm{C}$, the bacteria formed a biofilm layer at the bottom. Biofilms cells were harvested by scraping off the biofilm.

Growth curves of wildtype and mutant strains were recorded using a Bioscreen $\mathrm{C}$ system (Oy Growth Curves Ab Ltd). Overnight cultures of the strains were 1:100 diluted into LB and TSB $1 / 20$ broth into three separate wells of a 100 well honeycomb plate (three biological repeats) and grown at $25{ }^{\circ} \mathrm{C}$ for $48 \mathrm{~h}$. The experiments were performed under continuous shaking conditions and the optical density $\left(\mathrm{OD}_{595}\right)$, reflecting the bacterial growth, was measured every $15 \mathrm{~min}$.

As an additional phenotypic biofilm-related assay we performed: Congo red (CR) morphotype formation tests. LB agar without $\mathrm{NaCl}$ supplemented with Congo red (40 $\mu \mathrm{g} / \mathrm{ml})$ and Coomassie brilliant blue $(20 \mu \mathrm{g} / \mathrm{ml})$ (CR agar 
plates), incubated at room temperature (i.e. $25^{\circ} \mathrm{C}$ ), was used to judge colony morphology and color (i.e. rdar morphotype formation), reflecting proper EPS production (mainly curli and cellulose for $S$. Typhimurium) [10].

\section{ChIP-chip analysis}

Salmonella strains were cultured under free-living TSB conditions (TSB 1/20, $200 \mathrm{rpm}, 25^{\circ} \mathrm{C}$ ) until an $\mathrm{OD}_{595}$ of 0.3 (ca. $2 \times 10^{8}$ cells) was reached (ca. 6 h). ChIP experiments were performed as previously described [35] on the fabR-M9 S. Typhimurium SL1344 strain (CMPG5825). ChIP-enriched DNA fragments were blunted and amplified via ligation-mediated (LM) PCR using PRO336 and PRO337 [42], and sent to NimbleGen Systems, Inc. ChIP samples were labelled with $\mathrm{Cy} 5$ and hybridized against a genomic DNA reference, labelled with $\mathrm{Cy} 3$, on $S$. Typhimurium LT2 whole genome tiling arrays. The arrays consisted of 387,000 unique 50 -mer probes covering the whole $S$. Typhimurium LT2 genome and pSLT plasmid with a moving window overlap of 12 bases [43]. Note that $S$. Typhimurium SL1344 does not contain the prophages Fels-1 and Fels-2 as compared to $S$. Typhimurium LT2 [44, 45]. Raw data were normalized by polynomial regression and $\log _{2}$ ratios of ChIP over reference ( $\log _{2}$ enrichment ratios) were calculated (Matlab). To identify FabR-bound enriched regions in the ChIP-chip data, peak detection was performed with Mpeak [46] using the $\log _{2}$ enrichment ratios as input and performing first 'simple cluster detection' and then 'peak detection'. All default parameter values were used, except for the 'minimum number of probes', which was set to 45 to account for the sonication process. Sonication produces DNA fragments of $800 \mathrm{bp}$ on average, such that the ensemble of all retrieved FabR-bound DNA fragments for a specific target gene span a region of approximately $1700 \mathrm{bp}$, corresponding to ca. 45 probes in our tiling array design. The default Mpeak $p$-value is 0.01. $\log _{2}$ enrichment ratios and identified peaks were visualized with SignalMap software (NimbleGen Systems, Inc.).

\section{Regulatory motif detection}

The promoter or intergenic regions of the confirmed FabR-bound target genes were screened for conserved sequences using MotifSampler [47]. The promoter regions were extracted from the $S$. Typhimurium SL1344 genome sequence, as available via the website of the Sanger Institute (U.K.) (http://www.sanger.ac.uk/Projects/Salmonella). The whole promoter ( $f a b A$, $f a b B$ and $y q f A$ ) or intergenic (hpaR/hpaG and $d d g /$ $y f d Z)$ regions were used as seeds for the algorithm, with default parameters and motif widths ranging between 8 and $15 \mathrm{bp}$.

\section{ChIP-qPCR analysis}

ChIP was repeated for two biological replicates of both fabR-M9 (CMPG5825, ChIP samples) and wildtype cultures (mock ChIP samples) grown under free-living TSB conditions (TSB 1/20, $200 \mathrm{rpm}, 25^{\circ} \mathrm{C}, 6 \mathrm{~h}$ ) as described above, and the enrichment of the promoter regions was assessed by qPCR with $d n a G$ [48] as endogenous control. The enrichment ratios of ChIP over mock ChIP samples were calculated as $R Q=2^{-(\Delta C t \text { ChIP }-\Delta C t \text { mock ChIP })}$, in which $\Delta \mathrm{Ct}_{\text {ChIP }}$ is $\mathrm{Ct}_{\text {gene test }}-\mathrm{Ct}_{\text {dnaG }}$ for the ChIP samples and $\Delta \mathrm{Ct}_{\text {mock ChIP }}$ is $\mathrm{Ct}_{\text {gene test }}-\mathrm{Ct}_{\text {dnaG }}$ for the mock ChIP samples. Additionally, cells were cultured under LB conditions (LB, $200 \mathrm{rpm}, 25^{\circ} \mathrm{C}$ ) until an $\mathrm{OD}_{595}$ of 0.7 (ca. $5 \times 10^{8}$ cells) was reached (ca. 5-6 h)) and biofilm conditions (TSB $1 / 20,25{ }^{\circ} \mathrm{C}, 48 \mathrm{~h}$ at the bottom of petri dishes as described above). qPCR reactions were performed on $1 \mu \mathrm{l}$ of ChIP and mock ChIP-enriched DNA and serial dilutions of input DNA on the StepOnePlus (ABI) using PowerSYBR Green PCR Master Mix (Applied Biosystems), according to the manufacturer's instructions. ChIP-qPCR primers (Additional file 1: Table S2) were designed with Primer Express 3.0 (Applied Biosystems). All reactions were at least performed in triplicate. Amounts of PCR product in the ChIP and mock ChIP samples were determined using the StepOne ${ }^{\mathrm{m}}$ (version 2.1) and DataAssist ${ }^{\text {tw }}$ (version 2.0) software from Applied Biosystems.

\section{Transcriptome microarray analysis and qRT-PCR validation}

S. Typhimurium SL1344 wildtype and CMPG5624 $(\triangle f a b R)$ were cultured as discussed above under freeliving TSB conditions. Samples were treated with $1 / 5$ volume ice-cold phenol:ethanol mixture (5:95) and transferred to a microcentrifuge tube which was immediately frozen in liquid nitrogen and stored at -80 ${ }^{\circ} \mathrm{C}$. Total RNA was isolated with the Qiagen RNeasy mini kit according to the manufacturer's protocol. Contaminating genomic DNA was removed from the RNA samples with Turbo DNA-free (Ambion). Removal of DNA was checked by PCR. Prior to labeling, the concentration of total RNA was determined by measuring the $\mathrm{A}_{260}$ with a NanoDrop spectrophotometer (ND-1000). RNA was labeled with Cy5 and Cy3 by reverse transcription [9]. Hybridizations were performed in color flip on $S$. Typhimurium arrays containing 70-mer oligos representing all LT2 annotated genes (Operon), spotted in duplicate on CodeLink Activated slides (Amersham Biosciences), as previously reported [35]. Data were Loess normalized with the LIMMA BioConductor package without performing a background correction. Differentially expressed genes were detected by $t$-test with multiple testing correction ( $p$-value $<0.02$ and absolute fold change $>1.3$ ). 
Expression of a selected number of genes was additionally assayed through qRT-PCR (two biological repeats), as previously discussed [40]. The used qRT-PCR primers are listed in Additional file 1: Table S2. cDNA was prepared starting from $100 \mathrm{ng}$ of DNase-treated RNA extracted from cells cultured under the conditions as specified above. Normalization of the target gene's expression was performed using dnaG, gyrB, recA, rfaH and $r r s G$ as endogenous controls using the DataAssist ${ }^{\mathrm{Tm}}$ (version 2.0) software package from Applied Biosystems.

\section{Results}

\section{FabR involvement in S. Typhimurium biofilm formation}

Mutant screening revealed a S. Typhimurium SL1344 fabR deletion mutant (CMPG5624) to show an impaired biofilm formation at 16,25 and $30{ }^{\circ} \mathrm{C}$ when grown in TSB $1 / 20$ for $48 \mathrm{~h}$. Different temperatures were tested because the regulation of biofilm formation in Salmonella is known to be strongly temperature dependent [4]. The observed biofilm defect could be complemented by introducing fabR in trans (pCMPG5678) (Fig. 1). The complementation experiment also indicated that balanced $f a b R$ expression -with respect to expression level and/or kinetics- is necessary for proper biofilm formation. Indeed, the complemented $\triangle f a b R$ mutant, in which $f a b R$ expression is driven by a constitutive promoter, shows increased biofilm formation relative to the wildtype, especially at $25{ }^{\circ} \mathrm{C}$. Since for the $\Delta f a b R$ mutant no defect in planktonic growth (in TSB $1 / 20$ nor LB) or CR morphotype formation (reflecting rdar morphotype expression) [10] was observed (data not shown), it is unlikely that fabR deletion affects biofilm formation through an interference with respectively its growth characteristics or EPS (cellulose or curli) production.

\section{Genome-wide identification of direct FabR target genes by ChIP-chip}

ChIP-chip analysis was performed to unravel the direct, in vivo transcriptional target network of the $S$. Typhimurium SL1344 FabR regulator on a genome-wide scale (GEO record: GSE52877). It was performed using CMPG5825, a $S$. Typhimurium SL1344 strain expressing a 9xMyc-tagged FabR protein from the native fabR chromosomal locus, on S. Typhimurium SL1344 whole genome tiling arrays, as described in Materials and Methods. The ChIP-chip experiment was performed for cells cultured for $6 \mathrm{~h}$ under free-living TSB conditions (TSB 1/20, $200 \mathrm{rpm}, 25^{\circ} \mathrm{C}$ ). Several factors needed for initiation of biofilm formation are already present in planktonic cells prior to attachment [49]. There is thus a strong possibility that the biofilm defect of the $\Delta f a b R$ mutant is caused by changes in these factors within planktonic cells.

In this analysis we identified 30 individual peaks reflecting putative FabR-DNA-binding sites. Peaks located within coding sequences (STM0955, STM1333, STM1334.c, STM1336, STM2108, STM2249, STM2250, STM2419, STM2617, STM3281, STM3814, and STM3815) were for the time being discarded from further analysis. After merging individual peaks in the same genomic regions, our analysis identified twelve possible FabR targets (Table 2). These twelve putative FabR targets (i.e. intergenic or promoter regions corresponding to one or two gene(s), respectively), are involved in different processes, as indicated in Table 2. Surprisingly, FabR

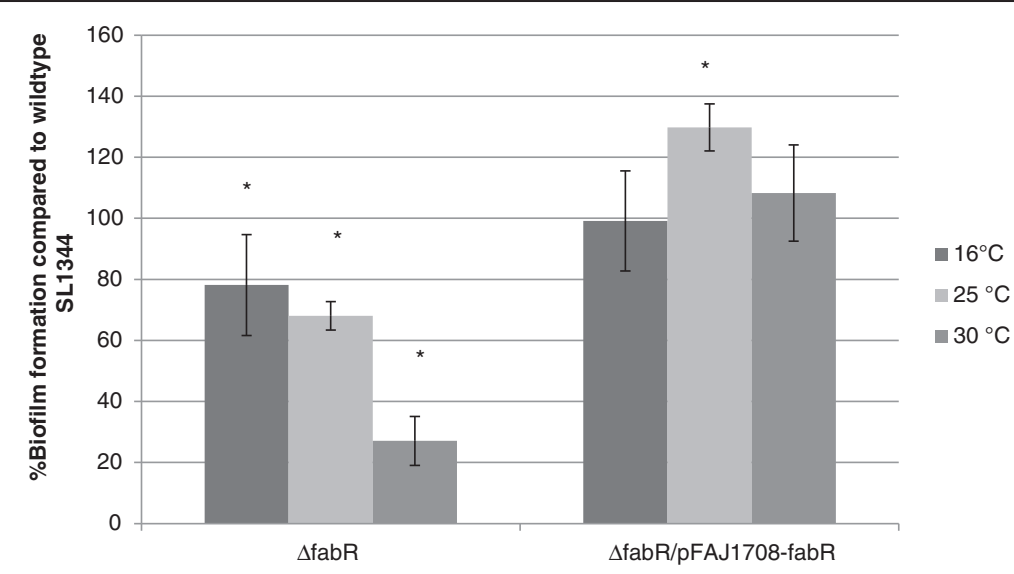

Fig. 1 Biofilm formation by an isogenic fabR mutant. The level of biofilm formation at the indicated temperatures is expressed as a percentage of wildtype SL1344 biofilm formation at the respective temperatures. The data are representative of three independent experiments $(n=3)$, with at least 4 replicates each. The error bars denote standard deviations between the independent experiments. For each temperature a one-sample $t$-test was performed to compare the mean biofilm formation of the mutant (expressed as a percentage of wildtype SL1344 biofilm formation) to $100 \%$. Significant changes ( $p$-value $<0.05$ ) in the level of biofilm formation as compared to the wildtype at the same temperature are indicated with an asterix $(*)$. SL1344: S. Typhimurium SL1344 wildtype strain; $\triangle f a b R$ : S. Typhimurium SL1344 $\triangle f a b R$ mutant (CMPG5624); $\triangle$ fabR-pFAJ1708-fabR trans complemented S. Typhimurium SL1344 $\triangle$ fabR mutant (pCMPG5678/CMPG5624) 
Table 2 Putative FabR targets identified using ChIP-chip

\begin{tabular}{|c|c|c|c|c|}
\hline Gene ID & Name $^{a}$ & Possible FabR Target $^{\mathrm{b}}$ & Function $^{\mathrm{a}}$ & Functional Class ${ }^{\mathrm{b}}$ \\
\hline STM1100 & hpaR $(<)$ & IR hpaR-hpaG & 4-Hydroxyphenylacetate catabolism & 1 \\
\hline STM1101 & hpaG (>) & & 4-Hydroxyphenylacetate catabolism & 1 \\
\hline STM1336 & $\operatorname{rp} / T(>)$ & P phes & 50 S ribosomal protein $L 20$ & 2 \\
\hline STM1337 & phes $(>)$ & & Phenylalanyl-tRNA synthetase subunit alpha & 3 \\
\hline STM2378 & $f a b B(<)$ & IR fabB-STM2379 & 3-Oxoacyl-(acyl carrier protein) synthase I & 4 \\
\hline STM2379 & STM2379 (>) & & 5-Methylaminomethyl-2-thiouridine methyltransferase & 5 \\
\hline STM2401 & $d d g(>)$ & $\mathrm{DR} d d g-y f d z$ & Lipid A biosynthesis palmitoleoyl acyltransferase & 6 \\
\hline STM2402 & $y f d Z(<)$ & & Aminotransferase & 7 \\
\hline STM2415 & $\operatorname{glt} X(<)$ & IR gltX-valU & Glutamyl-tRNA synthetase & 3 \\
\hline STM2416 & $\operatorname{valu}(>)$ & & tRNA & 3 \\
\hline STM2615 & STM2615 (<) & P STM2615 & tRNA & 3 \\
\hline STM2616 & STM2616 (<) & & Antirepressor-like protein & 8 \\
\hline STM2616 & STM2616 (<) & P STM2616 & Antirepressor-like protein & 8 \\
\hline STM2617 & STM2617 (<) & & Antiterminator-like protein & 8 \\
\hline STM2989 & $\operatorname{met} Z(>)$ & P metW & tRNA & 3 \\
\hline STM2990 & metW $(>)$ & & tRNA & 3 \\
\hline STM3049 & yqfa $(<)$ & P yqfA & Putative hemolysin & 6 \\
\hline STM3050 & yqfB $(<)$ & & Hypothetical protein & 5 \\
\hline STM3289 & $\operatorname{met} Y(<)$ & IR metY-argG & tRNA & 3 \\
\hline STM3290.S & $\arg G(>)$ & & Argininosuccinate synthase & 7 \\
\hline STM4148 & $\operatorname{nusG}(>)$ & P rplK & Transcription antitermination protein & 8 \\
\hline STM4149 & $r p / K(>)$ & & 50 S ribosomal protein $L 11$ & 2 \\
\hline STM4150 & $\operatorname{rp} / A(>)$ & PrplJ & 50 S ribosomal protein $\mathrm{L} 1$ & 2 \\
\hline STM4151 & $r p / J(>)$ & & $50 S$ ribosomal protein $L 10$ & 2 \\
\hline
\end{tabular}

${ }^{a}$ STM numbers, gene names, genomic orientation ( $<$ and $>$ indicating minus and positive strand, respectively) and gene functions are taken from NCBI Refseq NC_003197 [86] and results are sorted according to their STM numbers

${ }^{\mathrm{b}}$ The mentioned genes belong to the following functional classes: (1) Carbon compound degradation, (2) Ribosomal protein synthesis and modification, (3) Aminoacyl tRNA metabolism, (4) Fatty acid metabolism, (5) Conserved hypothetical protein, (6) Membrane homeostasis, (7) Amino acid biosynthesis, (8) RNA synthesis, RNA modification and DNA transcription

${ }^{\mathrm{b}} \mathrm{IR}$ indicates that both genes are possible FabR targets since the FabR binding region was situated in the intergenic region between the two mentioned divergently transcribed genes and hence contains the (putative) promoters of both genes. $P$ points at the (putative) promoter region of the mentioned gene since the intergenic region identified during the ChIP-chip analysis was situated between two genes transcribed in the same direction and hence only contains the (putative) promoter of this gene. DR indicates that probably none of the identified genes is a putative FabR target since both adjacent genes are convergently transcribed respective to the retained intergenic FabR binding region

appears to bind in the promoter regions of three copies of the $S$. Typhimurium initiator tRNA genes that encode tRNA ${ }^{\text {fMet }}$ [50], according to the predicted FabR binding peaks. The putative FabR-DNA-binding sites situated in the promoter regions of ribosomal genes and tRNAs were, however, not the focus of our further investigation. Of the five remaining putative targets, two are known FabR targets in E. coli $[29,51,52]$ : fabB, encoding $\beta$-ketoacyl-ACP synthase catalysing the ratelimiting and essential reaction during the unsaturated fatty acid biosynthesis [53], and $y q f A$, encoding an inner membrane protein of the hemolysin 3 family with putative oxidoreductase function [54], while the three others are for the first time linked to in vivo FabR binding. These novel FabR target sites include (i) the intergenic region between the divergently transcribed $h p a R$ and $h p a G$ genes, both involved in aromatic catabolism (4- and 3-hydroxyphenylacetate degradation) [55, 56]; (ii) the intergenic region between divergently transcribed met $Y$ and $\operatorname{argG}$ encoding a tRNA and argininosuccinate synthase, respectively; and (iii) the intergenic region between the convergently transcribed genes $d d g$, involved in lipid A biosynthesis, and $y f d Z$, encoding an aminotransferase. The latter genomic organization makes it, however, unlikely for FabR to exert any regulatory function in this region.

Further validation of the ChIP-chip results was obtained by ChIP-qPCR. As can be seen in Fig. 2, the metY/argG intergenic region appeared not to be upregulated in the ChIP-qPCR analysis, suggesting that 


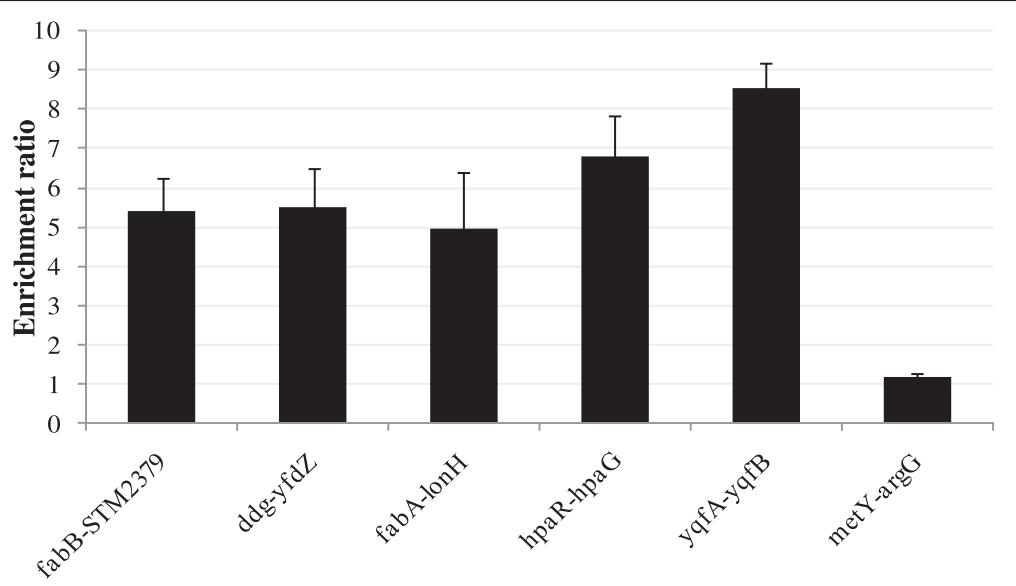

Fig. 2 ChIP-qPCR validation of the ChIP-chip data. The validation of the ChIP-chip results by ChIP-qPCR analysis was performed as elaborated in Materials \& Methods with dnaG as endogenous control. Values represent enrichment ratios of ChIP over mock ChIP samples, generated under free-living TSB conditions, and are averages of triplicate ChIP-qPCRs. The enrichment ratios of ChIP over mock ChIP samples were calculated as

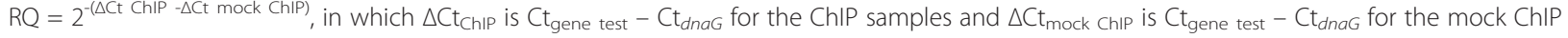
samples. The presented values are representative for two biological repeats and standard deviations of the three technical repeats are indicated

it might represent an artifact (i.e. false positive) of the ChIP-chip analysis [33]. ChIP-qPCR for the verified targets was also performed on ChIP samples taken under LB and biofilm conditions. FabR appeared to bind the investigated promoter regions under these different environmental conditions as well (Additional file 1: Figure S1), substantiating the direct, in vivo binding of $S$. Typhimurium FabR.

Next to the two known $E$. coli targets, FabR is also known to control the expression of fabA in E. coli by binding to its promoter region $[29,52]$. Although not detected on the tiling array, we could show binding of FabR to the $f a b A$ promoter by ChIP-qPCR, under TSB, LB and biofilm conditions. In $S$. Typhimurium, this represents the first evidence for direct in vivo binding of FabR to the $f a b B, f a b A$ and $y q f A$ promoters.

\section{Sequence conservation in the promoter region of direct FabR regulon members}

The FabR targets $f a b A, f a b B$ and $y q f A$ were previously identified in $E$. coli starting from a common motif identified using an in silico phylogenetic footprinting analysis [51]. Subsequent verification of the biological significance of this motif for FabR binding indicated the importance of the sequences flanking the motif for FabR binding in the absence -but not in the presence- of unsaturated thioesters, which are the native FabR ligands [29]. The sequence alignment of the originally identified degenerative binding palindrome upstream of their coding sequences in the case of $S$. Typhimurium SL1344, is depicted in Fig. 3a. To further validate that the newly in vivo identified targets are controlled by FabR, their putative intergenic regions were in silico screened for an overrepresented motif using the motif discovery algorithm MotifSampler [47]. More specifically, a de novo screening procedure was performed starting from the promoter and intergenic regions of the five ChIP-qPCR validated FabR targets. As shown in Fig. 3b, essential parts of the FabR palindromic recognition sequence were retained in all of them. Given the $2 \mathrm{bp}$ 'gap' of all other aligned sequences as compared to the hpaR/hpaG one, it seems reasonable to postulate that ACAnnTGTnnnnT constitutes the 'core' motif.

\section{Transcriptional profiling of a fabR mutant}

Since ChIP-chip data give information on the location of a regulator (i.e. direct binding), not on its functioning (i.e. not on the downstream effects this binding causes) and since promoter regions are often enriched by ChIPchip without being regulated by the transcription factor [33, 34, 57, 58], we complemented the ChIP-chip data with a transcriptomics study. Hereto, mRNA levels in the wildtype and the $\triangle f a b R$ mutant (CMPG5624) were compared at the same time point and under the same experimental conditions as used for the ChIP-chip experiment (GEO record: GSE52880). In view of a stringent selection, genes with an absolute fold change $>1.3$ and a $p$-value $<0.02$ were considered as significant. This threshold is acceptable since most regulatory responses in nature appear to function using low level changes as a kind of energy saving solution [59]. Of all genes on the array, respectively 179 genes (3.79\%) and 119 genes (2.52\%) were significantly down- and upregulated, in the $f a b R$ mutant as compared to the wildtype (functionally visualized in Fig. 4). A set of 12 of these differentially expressed genes was verified by qRT-PCR measurements and as can be seen in Fig. 5, the array data are overall in good agreement with the qRT-PCR data. 
a $\begin{array}{ll}\text { fabA } & \text { AGCGTACACGTGTTAGCT } \\ \text { fabB } & \text { GGCGTACAAGTGTACGCT } \\ \text { yqfA } & \text { AGCTAACATGTGTTCACT }\end{array}$ b

$\begin{aligned} \text { ddg } & \text { TCCCG--ACACTTGTAAGTT } \\ \text { fabA } & \text { AGCGT--ACACGTGTTAGCT } \\ \text { fabB } & \text { GGCGT--ACAAGTGTACGCT } \\ \text { hpaR } & \text { AGCTATGACATCTGTTT TAT } \\ \text { yqfA } & \text { AGCTA--ACATGTGTTCACT }\end{aligned}$

Fig. 3 Alignment of the putative FabR binding site in the ChIP-qPCR verified in vivo FabR targets. a Alignment of the already known E. coli FabR targets [51] in S. Typhimurium SL1344; b Alignment of the ChIP-chip identified and ChIP-qPCR verified FabR targets in S. Typhimurium SL1344. All alignments were performed using MotifSampler [47]. Sequences upstream of the coding sequences of the indicated genes were taken from the complete genome sequence of S. Typhimurium SL1344. These input sequences comprised the full intergenic region, i.e. the region between the coding sequence of the FabR target gene and the upstream coding sequence, with hpaR and $d d g$ indicating the hpaR/hpaG and $d d g / y f d z$ intergenic sequences, respectively. White letters with black background denote identical bases and black letters on a white background denote differing bases

The genes upregulated in the fabR deletion mutant were found to be strongly overrepresented in the groups of genes involved in general degradation processes (such as the fatty acid degradation genes $f a d A, f a d B$ and $f a d E$ ), fatty acid biosynthesis, biosynthesis/modification of ribosomal proteins and protein translation/modification, consistent with the putative binding sites identified during the ChIP-chip analysis. As expected, the direct targets $(f a b B$, $f a b A$ and $y q f A$ ) were significantly upregulated, confirming the direct repressor function of FabR on these genes in $S$.
Typhimurium (Table 3 and Additional file 1: Table S1). Moreover, combination of the transcriptomic and the ChIP-chip results provides the first experimental evidence that FabR directly binds to the $y q f A$ promoter and regulates its expression. This result contrasts with recent findings by Feng and Cronan, who observed that FabR binding to $y q f A$ was without physiological consequence in E. coli background (i.e. no regulatory role for FabR in regulating yqfA expression under their tested conditions) [29]. The data presented in Table 3 also indicate that FabR

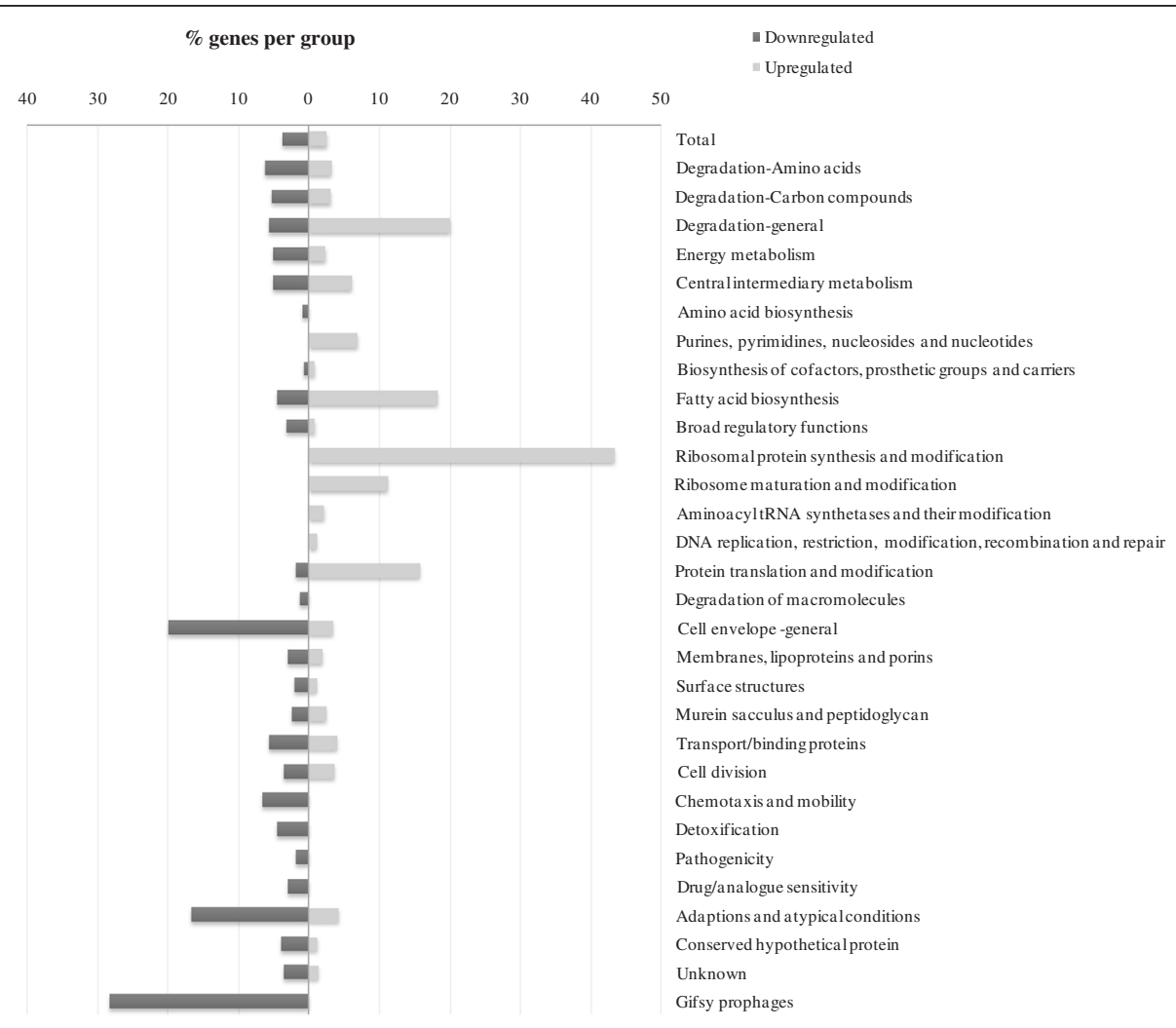

Fig. 4 Functional classification of the differentially expressed genes in the isogenic fabR deletion mutant as compared to the wildtype under free-living TSB conditions. The bars represent the percentage of genes belonging to each group that were altered for absolute expression > 1.3 fold with a $p$-value $<0.02$. The functional classes defined by the Welcome Trust Sanger Institute were used for this classification and the numbers behind each class represent the number of genes in this class 


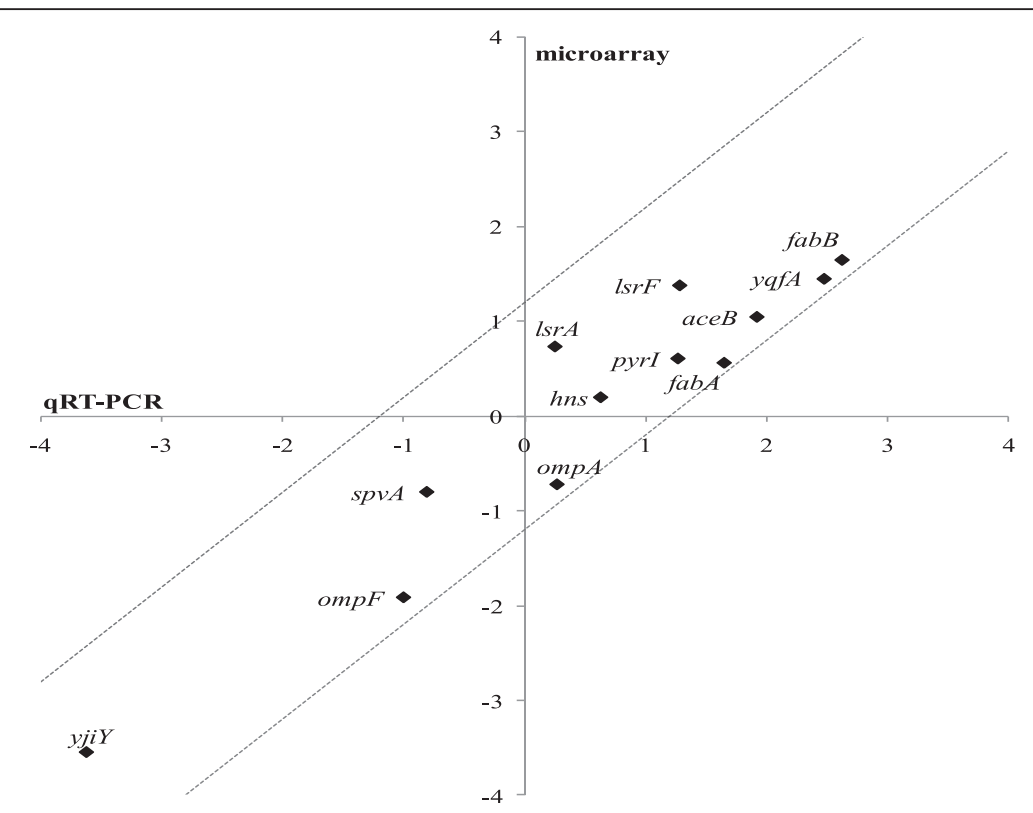

Fig. 5 Comparison between the microarray and qRT-PCR data. The expression of a number of genes was determined using qRT-PCR for the $S$. Typhimurium SL1344 wildtype and SL1344 $\triangle$ fabR mutant under free-living TSB conditions. The $\log _{2}$-transformed mean value of at least three qRT-PCR technical repeats (representative for each of the two assayed biological replicates) for each gene was plotted on the X-axis and compared to the respective $\log _{2}$-transformed microarray fold change (Y-axis). All depicted qRT-PCR tested genes had a $p$-value $<0.02$ under their respective microarray conditions and their GRT-PCR primers are listed in Additional file 1: Table S2. The dotted lines represent arbitral boundaries $(y=x+1.2$ and $y=x-1.2)$ between which the corresponding qRT-PCR and microarray results show good correspondence (i.e. not more than a 1.2 fold divergence on $\log _{2}$ scale) with $y=x$ being the ideal situation

is more dedicated towards $f a b B$ regulation as compared to $f a b A[29,31]$. The differential expression of other genes involved in the fatty acid metabolism (Table 3) might compensate for maintaining normal cellular fatty acid composition and energy homeostasis in the fabR mutant. Similar crosstalk between different components of the fatty acid metabolism (biosynthesis, degradation and uptake) in fatty acid regulatory null mutants has previously been described [31]. In this context, it was also not surprising to encounter an upregulated expression of the aceB-aceA-aceK operon in the fabR deletion mutant (Table 3 ). This operon encodes enzymes in the glyoxylate shunt and as such connects the TCA cycle and the fatty acid metabolism, both crucial processes in balancing cellular energy levels [60]. In addition, the whole $l s r R$ regulon [36] involved in AI-2 uptake and processing, was found to be significantly upregulated in a fabR mutant. Although still under investigation in S. Typhimurium [61-64], the LuxS synthesized AI-2 has previously been linked to biofilm formation [65]. The exact reason why these genes are upregulated in a $f a b R$ mutant, however, remains to be elucidated since no direct binding to the $l s r R$ promoter region was identified in the Chip-chip (and ChIP-qPCR, data not shown) analysis. The upregulation of the genes involved in ribosomal protein synthesis and modification (Table 3 and Fig. 4) is consistent with the putative binding of FabR to such genomic regions (Table 2). Although the
ChIP-chip and ChIP-qPCR experiments indicated FabR to bind to the hpaR-hpaG intergenic region, none of these genes were significantly upregulated in the $f a b R$ mutant.

The downregulated genes (Fig. 4, Additional file 1: Table S1) were found to be overrepresented in the classes of genes involved in cell-envelope structure (including the outer membrane protein-encoding genes $s p v A$, $n m p C$ (ompD), ompA, ompC, ompF and ompX) and adaptation processes (such as yji $Y$ encoding a carbon starvation protein and the $\operatorname{osm} B$ and $\operatorname{os} m C$ genes encoding osmotically inducible proteins), as well as lysogenic Gifsy prophage genes (encoding putative virulence factors [44, 45]) (Fig. 4). This activating effect of FabR most likely happens indirectly because none of the in vivo ChIP-chip identified target genes showed a downregulated expression in the $f a b R$ mutant relative to the wildtype strain.

\section{fabB is important for Salmonella biofilm formation}

Finally, we investigated whether the biofilm defect of a $f a b R$ mutant could be attributed to the observed enhanced expression of any of the direct targets $f a b A, f a b B$ or $y q f A$. Hereto we individually overexpressed these genes by respectively introducing plasmids pCMPG10118, pCMPG10119 and pCMPG5553 in the wildtype strain. In these plasmids the genes are cloned downstream of the constitutive nptII promoter. 
Table 3 Top-35 upregulated genes under free-living TSB conditions in the $\triangle f a b R$ mutant versus the wildtype

\begin{tabular}{|c|c|c|c|c|c|}
\hline Ranking $^{\text {a }}$ & $1 D^{b}$ & Name $^{\mathrm{b}}$ & Function $^{\mathrm{b}}$ & Fold $^{a}$ & Process $^{\mathrm{b}}$ \\
\hline 1 & STM2378 & $f a b B$ & 3-Oxoacyl-[acyl-carrier-protein] synthase I & 3.14 & Fatty acid metabolism \\
\hline 6 & STM3982 & fadA & $\beta$-subunit of the fatty acid-oxidizing multi-enzyme complex & 2.26 & \\
\hline 8 & STM3983 & $f a d B$ & a-subunit of the fatty acid-oxidizing multi-enzyme complex & 2.16 & \\
\hline 19 & STM0309 & yafH/fadE & Acyl-CoA dehydrogenase & 1.71 & \\
\hline 34 & STM0454 & ybaW/fadM & Long-chain acyl-CoA thioesterase III & 1.49 & \\
\hline 35 & STM1067 & $f a b A$ & D-3-hydroxydecanoyl-(acyl carrier-protein) & 1.48 & \\
\hline 2 & STM3049 & yqfA & Putative hemolysin & 2.73 & Membrane-related \\
\hline 31 & STM1254 & & Putative lipoprotein & 1.51 & \\
\hline 3 & STM4078 & yneB/lsrF & Putative fructose 1-6-phosphate aldolase & 2.60 & Al-2 metabolism \\
\hline 5 & STM4079 & yneC/lsrG & Isomerase for processing of phospho-Al-2 & 2.49 & \\
\hline 7 & STM4080 & IsrE & Putative ribulose-5-phosphate 3-epimerase & 2.24 & \\
\hline 10 & STM4077 & yneA/lsrB & $A B C$ transport protein, solute-binding component & 2.13 & \\
\hline 12 & STM4071 & & Hypothetical protein & 2.02 & \\
\hline 13 & STM4072 & ydeV/Isrk & Sugar kinase & 1.87 & \\
\hline 17 & STM4076 & ydeZ/IsrD & $A B C$ transporter, membrane component & 1.75 & \\
\hline 21 & STM4074 & ego/lsrA & ABC transporter ATP-binding protein & 1.66 & \\
\hline 23 & STM4075 & ydeY/IsrC & $A B C$ transporter permease protein & 1.64 & \\
\hline 4 & STM4184 & aceA & Isocitrate lyase & 2.50 & Glyoxylate metabolism \\
\hline 11 & STM4183 & $a c e B$ & Malate synthase A & 2.07 & \\
\hline 14 & STM4185 & acek & Isocitrate dehydrogenase kinase/phosphatase & 1.86 & \\
\hline 9 & STM3195 & $r i b B$ & 3,4-Dihydroxy-2-butanone 4-phosphate synthase & 2.14 & Riboflavin metabolism \\
\hline 15 & STM2935 & cys $D$ & ATP sulphurylase (ATP:sulphate adenyltransferase) & 1.79 & Sulphur metabolism \\
\hline 16 & STM3304 & $r p / U$ & $50 S$ ribosomal subunit protein L21 & 1.78 & Ribosomal protein synthesis and modification \\
\hline 22 & STM3303 & rpmA & $50 S$ ribosomal subunit protein L27 & 1.65 & \\
\hline 26 & STM3209 & $\operatorname{rps} U$ & $30 S$ ribosomal subunit protein S21 & 1.62 & \\
\hline 27 & STM3345 & $r p / M$ & $50 S$ ribosomal subunit protein L13 & 1.61 & \\
\hline 30 & STM3429 & $r p / X$ & $50 S$ ribosomal subunit protein L24 & 1.51 & \\
\hline 18 & STM2970 & sdac & Putative serine transporter & 1.72 & Transport processes \\
\hline 20 & STM1530 & & Putative outer membrane protein (truncation) & 1.68 & \\
\hline 28 & STM2444 & cys $P$ & Thiosulphate-binding protein precursor & 1.55 & \\
\hline 32 & STM1473 & $\mathrm{ompN}$ & Outer membrane protein & 1.51 & \\
\hline 33 & STM1452 & $y d g R$ & Putative proton/oligopeptide symporter & 1.49 & \\
\hline 24 & STM0307 & & Putative secreted protein & 1.64 & Pathogenicity \\
\hline 25 & STM0447 & tig & Trigger factor & 1.63 & Protein folding \\
\hline 29 & STM4459 & pyrl & Aspartate carbamoyltransferase regulatory subunit & 1.52 & Pyrimidine metabolism \\
\hline
\end{tabular}

aRank (\#) depends on the fold change with 1 being the gene with the greatest fold induction and 35 the one with the $35^{\text {th }}$ fold induction. Fold change represents the differential expression of the gene in the isogenic fabR deletion mutant (CMPG5624) according to the wildtype under free-living TSB conditions, as detected by $t$-test with multiple testing correction. ${ }^{b}$ STM numbers, gene names and gene functions are taken from the fully annotated S. Typhimurium LT2 genome (NCBI Refseq NC_003197) [86] and adapted according to recent literature (e.g. functions of the Isr genes were taken from [36, 87]). Results are sorted according to the functional classes (process) they belong to and with descending fold change in each class

As indicated in Fig. 6, overexpression of $f a b A$ and $y q f A$ does not result in a reduced biofilm formation. At $16{ }^{\circ} \mathrm{C}$ $y q f A$ overexpression even strongly increases biofilm formation, a phenotype which could prove interesting for further investigation. Overexpression of $f a b B$, however, does result in a decreased biofilm formation, indicating a role for $f a b B$ in mediating the effect of $f a b R$ on biofilm formation. At 25 and $30{ }^{\circ} \mathrm{C}$, the biofilm defect is less pronounced in the $f a b B$ overexpressing strain than in the $f a b R$ mutant, suggesting that next to $f a b B$ also other $f a b R$ targets might play a role in biofilm formation. At $16{ }^{\circ} \mathrm{C}$, however, the biofilm defect is much more pronounced 


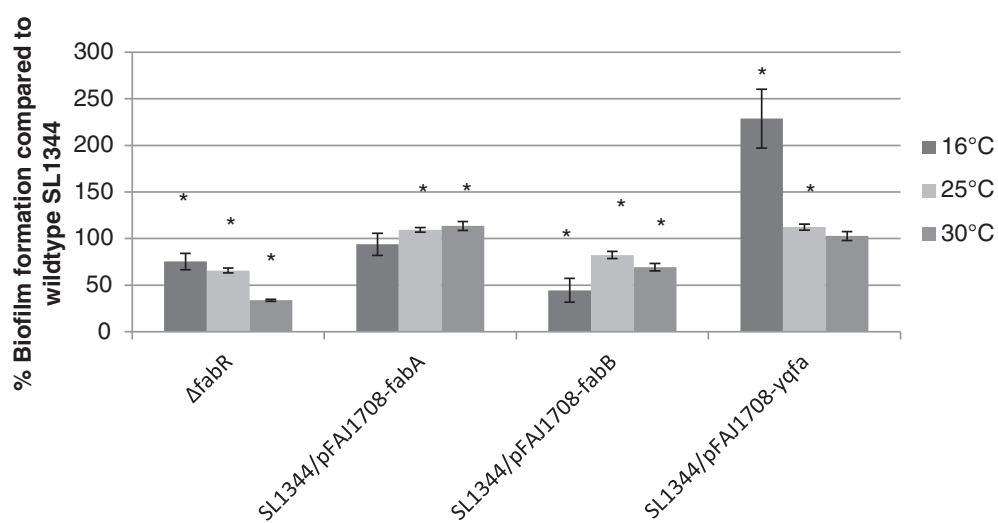

Fig. 6 Effect on biofilm formation of individually overexpressing $f a b A$, fabB and yafA. The level of biofilm formation at the indicated temperatures is expressed as a percentage of wildtype SL1344 biofilm formation. The data are representative of three independent experiments $(n=3)$, with at least 4 replicates each. The error bars denote standard deviations between the independent experiments. For each temperature a one-sample t-test was performed to compare the mean biofilm formation of the different strains (expressed as a percentage of wildtype SL1344 biofilm formation) to the wildtype level of $100 \%$. Significant changes ( $p$-value $<0.05$ ) in the level of biofilm formation as compared to the wild type at the same temperature are indicated with an asterix $\left(^{*}\right)$ SL1344: S. Typhimurium SL1344 wildtype strain; $\triangle$ fabR: S. Typhimurium SL1344 $\triangle f a b R$ mutant (CMPG5624); SL1344/pFAJ1708-fabA: S. Typhimurium SL1344 overexpressing fabA (pCMPG10118/SL1344); SL1344/pFAJ1708-fabB: S. Typhimurium SL1344 overexpressing fabB (pCMPG10119/SL1344); SL1344/pFAJ1708-yqfA: S. Typhimurium SL1344 overexpressing yqfA (pCMP5553/SL1344)

in the $f a b B$ overexpressing strain than in the $f a b R$ mutant. This could possibly be explained by the simultaneous, counteracting effects in the $f a b R$ mutant of $f a b B$ upregulation (reduced biofilm) and $y q f A$ upregulation (increased biofilm).

FabB plays a key role in UFA synthesis by catalyzing the elongation of the cis-3-decenoyl-ACP produced by FabA. Consistently, studies in $E$. coli indicated that $f a b B$ overexpression strongly increases UFA production $[29,66]$. Given the structural similarity of these UFA's with the DSF (diffusible signaling factor) family of compounds, which is known to induce biofilm dispersion [67], we hypothesized that the biofilm defect of the FabB overexpressing strain could be mediated by increased levels of free UFA's, acting as biofilm dispersing molecules. Adding a concentration range of different UFA's (cis-5dodecenoic acid, cis-9-hexadecenoic acid and cis-11hexadecenoic acid) to the growing biofilm, however, did not affect the amount of biofilm formed (data not shown).

\section{Discussion}

In this study, we showed that the unsaturated fatty acid biosynthesis regulator FabR is involved in $S$. Typhimurium biofilm formation. To unravel how this regulator impinges on Salmonella biofilm formation, a combinatorial high-throughput approach, combining ChIP-chip with transcriptomics, was applied. Highthroughput ChIP-chip analysis allowed the identification of in vivo FabR binding sites reflecting potential target genes (Table 2). Firstly, all the previously identified E. coli FabR transcriptional target genes ( $f a b A$, $f a b B$ and $y q f a$ ) were shown to be direct $S$. Typhimurium FabR targets as well, validating our approach.
Direct binding to the promoter region of $f a b A$, however, was only observed using ChIP-qPCR. The failure to detect FabR binding to this site by the ChIP-chip technique could be due to the sensitivity of the tiling array and/or stringency during the hybridization process [68] or the failure to randomly amplify this specific genomic region $[69,70]$. Secondly, ChIP-chip also identified some new, direct FabR targets, i.e. the intergenic regions between $h p a R / h p a G$ and $d d g / y d f Z$. The latter two genes ( $d d g$ and $y f d Z$ ) are, in contrast to hpaR and hpaG, convergently transcribed, making it unlikely for FabR to exert any direct regulatory function on their expression. Thirdly, our ChIP-chip analysis also identified a number of yet putative FabR target genes (e.g. genes involved in tRNA metabolism, ribosome synthesis and translation). These might also represent real in vivo FabR targets. Together, these data indicate that FabR only has a very limited regulon under the tested conditions. This contrasts to the broad regulon of global transcriptional regulators such as H-NS (ca. 745 direct target genes) [43] or FNR (ca. 100 direct target genes) [71] and the larger regulon of more specific regulators such as the invasion regulator HilA (ca. 20 direct target genes) [35].

Combining the ChIP-chip results with transcriptomics data provided information on the biological relevance of FabR binding for the identified regions. This combinatorial approach provides the first experimental evidence that FabR directly binds to and regulates the expression of $y q f A$, whereas for $E$. coli only evidence for binding has been given [29]. Moreover, we were able to extrapolate the previously observed higher tendency of FabR towards $f a b B$ regulation as compared to $f a b A$, from $E$. coli to $S$. Typhimurium [29, 31]. Although the 
ChIP-chip and ChIP-qPCR experiments indicated FabR to bind to the hpaR-hpaG intergenic region, none of these genes were significantly upregulated in the $f a b R$ mutant. Several possible explanations have been reported before for this observed lack of correlation between ChIP-chip and transcriptomics results (e.g. $[57,58])$. Some of these might also explain transcription factor binding to the intergenic region between convergently transcribed genes (such as FabR binding between $d d g / y d f Z$ ). In brief, the transcription factors might play other roles than regulating transcription or only have a minor impact on transcription levels. The binding sites may either serve as storage sites buffering the free pool of regulators, or have no physiological role under the given conditions, and/or depend on the presence or absence of other factors. Indeed, occupancy of a promoter region by a transcriptional regulator can be a necessary but not a sufficient condition for its transcriptional activity. In this respect, it was shown that FabR binding to the well-known $f a b A$ and $f a b B$ targets does not necessarily require unsaturated thioester ligands, but is enhanced in their presence $[29,52]$. An additional substantiation that the newly identified FabR targets are not just false positives was generated using in silico motif detection. Indeed, the hpaR/hpaG and $d d g / y f d Z$ intergenic regions shared a common consensus motif with the previously identified FabR targets. The degeneracy of the retrieved motif (and the above mentioned 'gap'), however, probably limit(s) the use of in silico prediction algorithms based on sequence data alone to map the FabR regulon.

As we found a $f a b B$ overexpressing strain to partly mimic the biofilm defect of the $f a b R$ mutant, the effect of FabR on biofilms can be attributed at least partly to the observed enhanced expression of its direct target $f a b B$. FabB plays a key role in UFA synthesis by catalyzing the elongation of the cis-3-decenoyl-ACP produced by FabA. In E. coli FabB overproduction has been shown to increase the synthesis of UFA's and to enhance the UFA contents of membrane phospholipids [29, 66]. The observation that a $f a b A$ overexpressing strain does not show this biofilm defect, although FabA and FabB catalyze two subsequent steps in the same pathway, can be explained by the assumption that FabB catalyses the rate limiting step. Indeed, in E. coli FabA overproduction was shown to increase the levels of SFA moieties rather than the levels of UFA's, an effect that was found to be nullified when both FabA and FabB were overproduced. This indicates that FabB is the limiting step in UFA synthesis and any excess cis-3-decenoyl-ACP produced by FabA would be diverted to the saturated fatty acid synthetic pathway [72]. Different, yet elusive, links between UFA synthesis and biofilm formation can be inferred. Firstly, the alterations in membrane fatty acid composition potentially impact on surface properties (roughness, cell surface charge, hydrophobicity, exposure of certain proteins, etc.) and biofilm formation. Membrane fluidity was indeed demonstrated to be essential in controlling swarming, a multicellular behaviour related to biofilm formation [73], and a biofilm phenotype-specific shift in membrane fatty acid composition has already been reported for $S$. Enteritidis [74]. Furthermore, fatty acids were also encountered in the EPS fraction of rdar-expressing S. Enteritidis strains [75]. Secondly, energy homeostasis, partly dependent on cellular fatty acid metabolism, has also been correlated with the energy-consuming Salmonella biofilm formation process [76]. Consistent with this, we not only noticed differential regulation of fatty acid-related genes, but also an alteration of the glyoxylate metabolism. Thirdly, as the UFA's synthesized by FabA and FabB show high similarities with DSFs (diffusible signaling factors), a known class of biofilm dispersing compounds [67], an alternative potential mechanism through which FabR could impact on biofilm formation is by increasing the levels of free UFA's acting as biofilm dispersing molecules. However, as we found that exogenous addition of different UFA's did not affect biofilm formation, this role of FabR in biofilm signaling is unlikely.

Next to UFA biosynthesis, a number of other processes regulated by FabR could possibly contribute to the effect of FabR on biofilm formation. ChIP-chip and microarray analysis indicated a direct FabR binding to and upregulation of ribosomal genes. The finding of Boehm et al. that ribosomal stress induces $E$. coli biofilm formation suggests a possible role for ribosome overexpression in biofilm reduction [77]. Also, direct links between Salmonella biofilm formation and genes downregulated in a $f a b R$ deletion mutant, such as ompA [64], ompC [78], отрX [79], osmB [80], osm C [79], sseI [78], have previously been identified, making them potential targets through which FabR could act on biofilm formation. Several of these repressed genes encode outer membrane proteins (ompA, ompC, ompX, osmB). Salmonella mutants in ompA and ompC have been shown to be deficient in biofilm formation on polystyrene and cholesterol-coated surfaces respectively [64, 78], whereas the expression of $\mathrm{omp} X$ and osm $C$ has been shown to be activated within Salmonella biofilms [79]. These outer membrane proteins are important for biofilm formation possibly because they mediate electrostatic interactions between salmonellae and the surface, promote overall biofilm health e.g. as nutrient channels, or have regulatory functions within biofilms [81].

\section{Conclusions}

In conclusion, we have shown that FabR is involved in Salmonella biofilm formation. In addition, we have illustrated that $S$. Typhimurium FabR has a limited regulon 
by combining ChIP-chip analysis with dedicated expression analysis. It directly controls the expression of $f a b A, f a b B$ and $y q f A$ by direct binding to their promoter regions. This confirms current knowledge generated in E. coli, but is the first evidence for the direct regulation of these genes by FabR in $S$. Typhimurium. Moreover, novel direct FabR targets were identified. FabB overexpression was shown to partly mimic the biofilm defect of the $f a b R$ mutant, indicating that the effect of FabR on biofilm formation can be attributed at least partly to its effect on $f a b B$ expression. Exploitation of the expression analysis data, allowed us to put forward some additional putative targets (direct and indirect) through which FabR might impact on biofilm formation. Overall, our results point at the importance of FabR and UFA biosynthesis in Salmonella biofilm formation and their role as potential targets for biofilm inhibitory strategies.

\section{Additional file}

Additional file 1: Supplementary material. (DOCX $66 \mathrm{~kb}$ )

\section{Competing interests}

The authors declare that they have no competing interests.

\section{Authors' contributions}

$\mathrm{KH}$ carried out the phenotypic assays, microarray analysis, ChIP-chip analysis, and (ChIP-)aPCR analysis and drafted the manuscript. SR, GK and DDC participated in the execution of the phenotypic assays, microarray analysis, ChIP-chip analysis, and (ChIP-)qPCR analysis. IT and KM performed the bioinformatics analysis of the microarray and ChIP-chip data and the footprinting analysis. JV, SDK and HS conceived of the study, and participated in its design and coordination and helped to draft the manuscript. All authors read and approved the final manuscript.

\section{Acknowledgements}

The authors thank Ms. A. De Weerdt for excellent technical assistant, Dr. C. Fierro for assistance in data processing, and Dr. S. Robijns for valuable discussions.

\section{Funding}

$\mathrm{KH}$ was a research assistant of the FWO-Vlaanderen at the time of the experiments and SR was a research assistant of the IWT-Vlaanderen. HS is and SDK was a post-doctoral researcher of the FWO-Vlaanderen. This research was supported by projects GOA/08/011 and IDO/11/008 of the KU Leuven Research Fund, by the Institute for the Promotion of Innovation through Science and Technology in Flanders under grant IWT-SBO 120050 (NEMOA) and the European Commission's Seventh Framework Programme (FP7/2007(2013) under the grant agreement COATIM (Project no. 278425)

\section{Author details}

${ }^{1}$ Department of Microbial and Molecular Systems, Centre of Microbial and Plant Genetics, Katholieke Universiteit Leuven, Kasteelpark Arenberg 20, 3001 Leuven, Belgium. ${ }^{2}$ Platform Biotechnology and Molecular Biology, Scientific Institute of Public Health (WIV-ISP), Brussels, Belgium.

Received: 21 April 2015 Accepted: 8 January 2016

Published online: 22 March 2016

\section{References}

1. Costerton JW. Introduction to biofilm. Int J Antimicrob Agents. 1999;11: 217-21. discussion 237-219.

2. Davies D. Understanding biofilm resistance to antibacterial agents. Nat Rev Drug Discov. 2003;2:114-22.
3. Joseph B, Otta SK, Karunasagar I, Karunasagar I. Biofilm formation by salmonella spp. on food contact surfaces and their sensitivity to sanitizers. Int J Food Microbiol. 2001;64:367-72.

4. Steenackers, H.P., Hermans, K., Vanderleyden, J. and De Keersmaecker, S.C. (2012) Salmonella biofilms: an overview on occurrence, structure, regulation and eradication. Food Res Int. 45:502-531.

5. Stepanovic S, Cirkovic I, Ranin L, Svabic-Vlahovic M. Biofilm formation by Salmonella spp. and Listeria monocytogenes on plastic surface. Lett Appl Microbiol. 2004;38:428-32

6. Bowen A, Fry A, Richards G, Beuchat L. Infections associated with cantaloupe consumption: a public health concern. Epidemiol Infect. 2006;134:675-85.

7. Prouty AM, Gunn JS. Comparative analysis of Salmonella enterica serovar Typhimurium biofilm formation on gallstones and on glass. Infect Immun. 2003;71:7154-8.

8. Jonas K, Tomenius H, Kader A, Normark S, Romling U, Belova LM, et al. Roles of curli, cellulose and BapA in Salmonella biofilm morphology studied by atomic force microscopy. BMC Microbiol. 2007;7:70.

9. Wang Q, Frye JG, McClelland M, Harshey RM. Gene expression patterns during swarming in Salmonella typhimurium: genes specific to surface growth and putative new motility and pathogenicity genes. Mol Microbiol. 2004;52:169-87.

10. Romling U, Sierralta WD, Eriksson K, Normark S. Multicellular and aggregative behaviour of Salmonella typhimurium strains is controlled by mutations in the agfD promoter. Mol Microbiol. 1998;28:249-64.

11. Simm R, Ahmad I, Rhen M, Le Guyon S, Romling U. Regulation of biofilm formation in Salmonella enterica serovar Typhimurium. Future Microbiol. 2014;9:1261-82.

12. Kader A, Simm R, Gerstel U, Morr M, Romling U. Hierarchical involvement of various GGDEF domain proteins in rdar morphotype development of Salmonella enterica serovar Typhimurium. Mol Microbiol. 2006;60:602-16.

13. Hengge R. Principles of c-di-GMP signalling in bacteria. Nat Rev Microbiol. 2009;7:263-73.

14. Zhang YM, Rock CO. Membrane lipid homeostasis in bacteria. Nat Rev Microbiol. 2008;6:222-33.

15. Ulrich AK, de Mendoza D, Garwin JL, Cronan Jr JE. Genetic and biochemical analyses of Escherichia coli mutants altered in the temperature-dependent regulation of membrane lipid composition. J Bacteriol. 1983;154:221-30.

16. Alvarez-Ordonez A, Fernandez A, Lopez M, Arenas R, Bernardo A. Modifications in membrane fatty acid composition of Salmonella typhimurium in response to growth conditions and their effect on heat resistance. Int J Food Microbiol. 2008;123:212-9.

17. Balamurugan S, Dugan ME. Growth temperature associated protein expression and membrane fatty acid composition profiles of Salmonella enterica serovar Typhimurium. J Basic Microbiol. 2010;50:507-18.

18. Suutari M, Laakso S. Microbial fatty acids and thermal adaptation. Crit Rev Microbiol. 1994:20:285-328.

19. Sampathkumar B, Khachatourians GG, Korber DR. Treatment of Salmonella enterica serovar Enteritidis with a sublethal concentration of trisodium phosphate or alkaline $\mathrm{pH}$ induces thermotolerance. Appl Environ Microbiol. 2004;70:4613-20.

20. Wang $Y$, Corrieu $\mathrm{G}$, Beal C. Fermentation $\mathrm{pH}$ and temperature influence the cryotolerance of Lactobacillus acidophilus RD758. J Dairy Sci. 2005:88:21-9.

21. Teixeira H, Goncalves MG, Rozes N, Ramos A, San Romao MV. Lactobacillic acid accumulation in the plasma membrane of Oenococcus oeni: a response to ethanol stress? Microb Ecol. 2002;43:146-53.

22. Guillot A, Obis D, Mistou MY. Fatty acid membrane composition and activation of glycine-betaine transport in Lactococcus lactis subjected to osmotic stress. Int J Food Microbiol. 2000:55:47-51.

23. Wang AY, Cronan Jr JE. The growth phase-dependent synthesis of cyclopropane fatty acids in Escherichia coli is the result of an RpoS(KatF)-dependent promoter plus enzyme instability. Mol Microbiol. 1994;11:1009-17.

24. Annous BA, Kozempel MF, Kurantz MJ. Changes in membrane fatty acid composition of Pediococcus sp. strain NRRL B-2354 in response to growth conditions and its effect on thermal resistance. Appl Environ Microbiol. 1999;65:2857-62.

25. Kiran MD, Annapoorni S, Suzuki I, Murata N, Shivaji S. Cis-trans isomerase gene in psychrophilic Pseudomonas syringae is constitutively expressed during growth and under conditions of temperature and solvent stress. Extremophiles. 2005;9:117-25. 
26. Chang YY, Cronan Jr JE. Membrane cyclopropane fatty acid content is a major factor in acid resistance of Escherichia coli. Mol Microbiol. 1999;33:249-59

27. Casadei MA, Manas P, Niven G, Needs E, Mackey BM. Role of membrane fluidity in pressure resistance of Escherichia coli NCTC 8164. Appl Environ Microbiol. 2002:68:5965-72.

28. Feng YJ, Cronan JE. Escherichia coli Unsaturated Fatty Acid Synthesis complex transcription of the fabA gene and in vivo identification of the essential reaction catalyzed by FabB. J Biol Chem. 2009;284:29526-35.

29. Feng Y, Cronan JE. Complex binding of the FabR repressor of bacterial unsaturated fatty acid biosynthesis to its cognate promoters. Mol Microbiol. 2011;80:195-218.

30. Magnuson K, Jackowski S, Rock CO, Cronan Jr JE. Regulation of fatty acid biosynthesis in Escherichia coli. Microbiol Rev. 1993;57:522-42.

31. Zhang YM, Marrakchi H, Rock CO. The FabR (YijC) transcription factor regulates unsaturated fatty acid biosynthesis in Escherichia coli. J Biol Chem. 2002;277:15558-65.

32. Ren B, Robert F, Wyrick JJ, Aparicio O, Jennings EG, Simon I, et al. Genomewide location and function of DNA binding proteins. Science. 2000;290:2306-9.

33. Waldminghaus T, Skarstad K. ChIP on Chip: surprising results are often artifacts. BMC Genomics. 2010;11:414.

34. De Keersmaecker SC, Thijs IM, Vanderleyden J, Marchal K. Integration of omics data: how well does it work for bacteria? Mol Microbiol. 2006;62:1239-50

35. Thijs IM, De Keersmaecker SC, Fadda A, Engelen K, Zhao H, McClelland M, et al. Delineation of the Salmonella enterica serovar Typhimurium HilA regulon through genome-wide location and transcript analysis. J Bacteriol. 2007;189:4587-96.

36. Thijs IM, Zhao H, De Weerdt A, Engelen K, De Coster D, Schoofs G, et al. The Al-2-dependent regulator LsrR has a limited regulon in Salmonella Typhimurium. Cell Res. 2010;20:966-9.

37. Sambrook J, Russel DW. Molecular cloning: a laboratory manual. 3rd ed. Cold Spring Harbor: Cold spring Harbor Laboratory Press; 2001.

38. Datsenko KA, Wanner BL. One-step inactivation of chromosomal genes in Escherichia coli K-12 using PCR products. Proc Natl Acad Sci U S A. 2000;97:6640-5

39. Knop M, Siegers K, Pereira G, Zachariae W, Winsor B, Nasmyth K, et al. Epitope tagging of yeast genes using a PCR-based strategy: more tags and improved practical routines. Yeast. 1999;15:963-72

40. Hermans K, Nguyen TL, Roberfroid S, Schoofs G, Verhoeven T, De Coster D et al. Gene expression analysis of monospecies Salmonella typhimurium biofilms using differential fluorescence induction. J Microbiol Methods. 2011;84:467-78.

41. Steenackers HP, Ermolat'ev DS, Savaliya B, De Weerdt A, De Coster D, Shah A, et al. Structure-activity relationship of 4(5)-aryl-2-amino-1H-imidazoles, N1-substituted 2aminoimidazoles and imidazo[1,2-a]pyrimidinium salts as inhibitors of biofilm formation by Salmonella Typhimurium and Pseudomonas aeruginosa. J Med Chem. 2011:54:472-84

42. Mueller PR, Wold B. In vivo footprinting of a muscle specific enhancer by ligation mediated PCR. Science. 1989;246:780-6.

43. Navarre WW, Porwollik S, Wang Y, McClelland M, Rosen H, Libby SJ, et al. Selective silencing of foreign DNA with low GC content by the H-NS protein in Salmonella. Science. 2006;313:236-8.

44. Figueroa-Bossi N, Uzzau S, Maloriol D, Bossi L. Variable assortment of prophages provides a transferable repertoire of pathogenic determinants in Salmonella. Mol Microbiol. 2001;39:260-71.

45. Frye JG, Porwollik S, Blackmer F, Cheng P, McClelland M. Host gene expression changes and DNA amplification during temperate phage induction. J Bacteriol. 2005;187:1485-92

46. Zheng M, Barrera LO, Ren B, Wu YN. ChIP-chip: data, model, and analysis. Biometrics. 2007:63:787-96.

47. Thijs G, Marchal K, Lescot M, Rombauts S, De Moor B, Rouze P, et al. A Gibbs sampling method to detect overrepresented motifs in the upstream regions of coexpressed genes. J Comput Biol. 2002;9:447-64.

48. Shin D, Groisman EA. Signal-dependent binding of the response regulators PhoP and PmrA to their target promoters in vivo. J Biol Chem. 2005;280:4089-94.

49. Janssens JC, Steenackers H, Robijns S, Gellens E, Levin J, Zhao H, et al. Brominated furanones inhibit biofilm formation by Salmonella enterica serovar Typhimurium. Appl Environ Microbiol. 2008;74:6639-48.

50. Nilsson Al, Zorzet A, Kanth A, Dahlstrom S, Berg OG, Andersson DI. Reducing the fitness cost of antibiotic resistance by amplification of initiator tRNA genes. Proc Natl Acad Sci U S A. 2006;103:6976-81.
51. McCue L, Thompson W, Carmack C, Ryan MP, Liu JS, Derbyshire V, et al. Phylogenetic footprinting of transcription factor binding sites in proteobacterial genomes. Nucleic Acids Res. 2001;29:774-82.

52. Zhu K, Zhang YM, Rock CO. Transcriptional regulation of membrane lipid homeostasis in Escherichia coli. J Biol Chem. 2009;284:34880-8.

53. Feng Y, Cronan JE. A New Member of the Escherichia coli fad Regulon: Transcriptional Regulation of fadM (ybaW). J Bacteriol. 2009;191:6320-8.

54. Miller EN, Jarboe LR, Yomano LP, York SW, Shanmugam KT, Ingram LO, Silencing of NADPH-dependent oxidoreductase genes (yghD and $\mathrm{dkgA}$ ) in furfural-resistant ethanologenic Escherichia coli. Appl Environ Microbiol. 2009;75:4315-23.

55. Galan B, Kolb A, Sanz JM, Garcia JL, Prieto MA. Molecular determinants of the hpa regulatory system of Escherichia coli: the HpaR repressor. Nucleic Acids Res. 2003;31:6598-609.

56. Galan B, Manso I, Kolb A, Garcia JL, Prieto MA. The role of FIS protein in the physiological control of the expression of the Escherichia coli meta-hpa operon. Microbiology. 2008;154:2151-60.

57. van Steensel B. Mapping of genetic and epigenetic regulatory networks using microarrays. Nat Genet. 2005;37(Suppl):S18-24.

58. Wade JT, Struhl K, Busby SJ, Grainger DC. Genomic analysis of protein-DNA interactions in bacteria: insights into transcription and chromosome organization. Mol Microbiol. 2007;65:21-6.

59. Wren JD, Conway T. Meta-analysis of published transcriptional and translational fold changes reveals a preference for low-fold inductions. Omics. 2006:10:15-27.

60. Fujita Y, Matsuoka H, Hirooka K. Regulation of fatty acid metabolism in bacteria. Mol Microbiol. 2007;66:829-39.

61. De Keersmaecker SC, Varszegi C, van Boxel N, Habel LW, Metzger K, Daniels $\mathrm{R}$, et al. Chemical synthesis of (S)-4,5-dihydroxy-2,3-pentanedione, a bacterial signal molecule precursor, and validation of its activity in Salmonella typhimurium. J Biol Chem. 2005;280:19563-8.

62. Jesudhasan PR, Cepeda ML, Widmer K, Dowd SE, Soni KA, Hume ME, et al. Transcriptome analysis of genes controlled by luxS/autoinducer-2 in Salmonella enterica serovar Typhimurium. Foodborne Pathog Dis. 2010;7:399-410

63. Prouty AM, Schwesinger WH, Gunn JS. Biofilm formation and interaction with the surfaces of gallstones by Salmonella spp. Infect Immun. 2002;70:2640-9.

64. Kint G, De Coster D, Marchal K, Vanderleyden J, De Keersmaecker SC. The small regulatory RNA molecule MicA is involved in Salmonella enterica serovar Typhimurium biofilm formation. BMC Microbiol. 2010;10:276.

65. Hardie KR, Heurlier K. Establishing bacterial communities by 'word of mouth': LuxS and autoinducer 2 in biofilm development. Nat Rev Microbiol. 2008;6:635-43.

66. Zhang F, Ouellet M, Batth TS, Adams PD, Petzold CJ, Mukhopadhyay A, et al Enhancing fatty acid production by the expression of the regulatory transcription factor FadR. Metab Eng. 2012;14:653-60.

67. Ryan RP, Dow JM. Communication with a growing family: diffusible signal factor (DSF) signaling in bacteria. Trends Microbiol. 2011;19:145-52.

68. Cho BK, Knight EM, Barrett CL, Palsson BO. Genome-wide analysis of Fis binding in Escherichia coli indicates a causative role for A-/AT-tracts. Genome Res. 2008;18:900-10.

69. Herring CD, Raffaelle M, Allen TE, Kanin El, Landick R, Ansari AZ, et al. Immobilization of Escherichia coli RNA polymerase and location of binding sites by use of chromatin immunoprecipitation and microarrays. J Bacteriol. 2005;187:6166-74.

70. Hanlon SE, Lieb JD. Progress and challenges in profiling the dynamics of chromatin and transcription factor binding with DNA microarrays. Curr Opin Genet Dev. 2004;14:697-705.

71. Grainger DC, Aiba H, Hurd D, Browning DF, Busby SJ. Transcription factor distribution in Escherichia coli: studies with FNR protein. Nucleic Acids Res 2007:35:269-78

72. Clark DP, DeMendoza D, Polacco ML, Cronan Jr JE. Beta-hydroxydecanoyl thio ester dehydrase does not catalyze a rate-limiting step in Escherichia coli unsaturated fatty acid synthesis. Biochemistry. 1983;22:5897-902.

73. Verstraeten N, Braeken K, Debkumari B, Fauvart M, Fransaer J, Vermant $J$, et al. Living on a surface: swarming and biofilm formation. Trends Microbiol. 2008;16:496-506.

74. Mangalappalli-IIlathu AK, Korber DR. Adaptive resistance and differential protein expression of Salmonella enterica serovar Enteritidis biofilms exposed to benzalkonium chloride. Antimicrob Agents Chemother. 2006;50:3588-96. 
75. Gibson DL, White AP, Snyder SD, Martin S, Heiss C, Azadi P, et al. Salmonella produces an O-antigen capsule regulated by AgfD and important for environmental persistence. J Bacteriol. 2006;188:7722-30.

76. White AP, Weljie AM, Apel D, Zhang P, Shaykhutdinov R, Vogel HJ, et al. A global metabolic shift is linked to Salmonella multicellular development. PLoS One. 2010;5, e11814.

77. Boehm A, Steiner S, Zaehringer F, Casanova A, Hamburger F, Ritz D, et al. Second messenger signalling governs Escherichia coli biofilm induction upon ribosomal stress. Mol Microbiol. 2009;72:1500-16.

78. Crawford RW, Reeve KE, Gunn JS. Flagellated but not hyperfimbriated Salmonella enterica serovar Typhimurium attaches to and forms biofilms on cholesterol-coated surfaces. J Bacteriol. 2010;192:2981-90.

79. Hamilton S, Bongaerts RJ, Mulholland F, Cochrane B, Porter J, Lucchini S, et al. The transcriptional programme of Salmonella enterica serovar Typhimurium reveals a key role for tryptophan metabolism in biofilms. BMC Genomics. 2009;10:599.

80. Garcia-Calderon CB, Casadesus J, Ramos-Morales F. Rcs and PhoPQ regulatory overlap in the control of Salmonella enterica virulence. J Bacteriol. 2007;189:6635-44.

81. Ma Q, Wood TK. OmpA influences Escherichia coli biofilm formation by repressing cellulose production through the CpxRA two-component system. Environ Microbiol. 2009:11:2735-46.

82. Hoiseth SK, Stocker BA. Aromatic-dependent Salmonella typhimurium are non-virulent and effective as live vaccines. Nature. 1981;291:238-9.

83. Cormack BP, Valdivia RH, Falkow S. FACS-optimized mutants of the green fluorescent protein (GFP). Gene. 1996:173:33-8.

84. Derbyshire KM, Willetts NS. Mobilization of the non-conjugative plasmid RSF1010: a genetic analysis of its origin of transfer. Mol Gen Genet. 1987;206:154-60.

85. Valdivia RH, Falkow S. Bacterial genetics by flow cytometry: rapid isolation of Salmonella typhimurium acid-inducible promoters by differential fluorescence induction. Mol Microbiol. 1996;22:367-78.

86. McClelland M, Sanderson KE, Spieth J, Clifton SW, Latreille P, Courtney L, et al. Complete genome sequence of Salmonella enterica serovar Typhimurium LT2. Nature. 2001;413:852-6.

87. Marques JC, Lamosa P, Russell C, Ventura R, Maycock C, Semmelhack MF, et al. Processing the Interspecies Quorum-sensing Signal Autoinducer-2 (Al-2) characterization of phospho-(S)-4,5-dihydroxy-2,3-pentanedione isomerization by LsrG protein. J Biol Chem. 2011;286:18331-43.

\section{Submit your next manuscript to BioMed Central and we will help you at every step:}

- We accept pre-submission inquiries

- Our selector tool helps you to find the most relevant journal

- We provide round the clock customer support

- Convenient online submission

- Thorough peer review

- Inclusion in PubMed and all major indexing services

- Maximum visibility for your research

Submit your manuscript at www.biomedcentral.com/submit 